\title{
Correlation between sialic acid levels in the synovial fluid and the radiographic severity of knee osteoarthritis
}

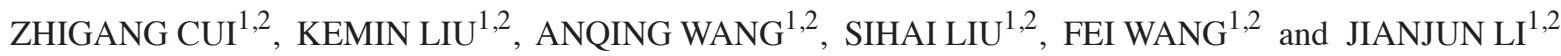 \\ ${ }^{1}$ Rehabilitation College, Capital Medical University, Beijing 100069; ${ }^{2}$ Department of Orthopedics and Rehabilitation, \\ China Rehabilitation Research Center, Beijing Charity Hospital, Beijing 100068, P.R. China
}

Received November 3, 2013; Accepted March 5, 2014

DOI: $10.3892 /$ etm.2014.1679

\begin{abstract}
Osteoarthritis (OA) is associated with the presence of inflammation. Sialic acid (SA), an acetylated derivative of neuraminic acid, is reported to be a useful biomarker of inflammation. The aim of the present study was to investigate the correlation between SA levels in the serum and synovial fluid (SF) and radiographic severity in patients with knee OA. A total of 234 patients with knee OA were recruited for the study, as well as 20 patients that had suffered a knee injury or fracture (without knee OA) and 160 healthy controls. Radiological grading of OA in the knee was conducted according to the Kellgren-Lawrence (KL) grading system. SA levels in the serum and SF were measured using Warren's thiobarbituric acid assay. The results demonstrated that knee OA patients exhibited significantly elevated levels of serum SA when compared with the healthy controls, and also significantly elevated levels of SF SA when compared with the knee fracture patients. Higher SA levels in the SF were identified in knee OA patients with KL grade 4 as compared with patients with KL grade 2 or 3 . In addition, OA patients of KL grade 3 had significantly higher SA levels in the SF as compared with patients with KL grade $2(\mathrm{P}<0.01)$. The SA levels in the SF of the knee OA patients positively correlated with the KL grades $(\mathrm{r}=0.353 ; \mathrm{P}<0.01)$. However, there was no significant correlation identified between serum SA levels and KL grade. Therefore, SA levels in the SF positively correlated with the radiographic severity of OA, thus, SA levels in the SF may serve as a biomarker for the progression of OA.
\end{abstract}

\section{Introduction}

Osteoarthritis (OA), a chronic degenerative joint disease, causes pain, stiffness, reduced motion, swelling, crepitus and disability. The disease is characterized by the progressive

Correspondence to: Dr Jianjun Li, Department of Orthopedics and Rehabilitation, China Rehabilitation Research Center, Beijing Charity Hospital, 10 Jiaomen Beilu, Beijing 100068, P.R. China

E-mail: lijianjunch@yeah.net

Key words: sialic acid, serum, synovial fluid, severity, osteoarthritis destruction of articular cartilage with joint-space narrowing, osteophyte formation, subchondral sclerosis and synovitis (1). A combination of risk factors, including aging, obesity, female gender, smoking, genetics, joint injury, mechanical and metabolic factors, have been shown to be involved in the pathophysiology of OA (2). However, the etiology and pathogenesis of OA remain poorly understood. Inflammation has been implicated in the pathogenesis of OA (3). Inflammatory markers, including interleukin-6, tumor necrosis factor- $\alpha$ (4) and C-reactive protein (5), in the serum and synovial fluid (SF) of OA patients have been reported to correlate with the radiographic severity of knee or hip OA.

Sialic acid (SA) is the terminal component at the non-reducing end of carbohydrate chains of glycoproteins and glycolipids (6). SA plays an important role in cell protection, fertilization, differentiation, adhesion, immunology and tumor development (7). Plasma SA is traditionally utilized as one of the biomarkers for the acute-phase response (8). Increased SA concentrations have been reported during inflammatory processes, possibly resulting from the elevated levels of richly sialylated acute-phase glycoproteins (9). In addition, elevated levels of SA have been reported to be associated with an increased risk of renal disease, diabetes, ovarian cancer and a variety of central nervous system disorders (10-12). A previous study demonstrated that patients with OA exhibited significantly elevated levels of serum SA when compared with healthy controls (13). Thus, it was hypothesized that SA may be involved in the mechanism underlying OA, and SA levels in the serum and SF may correlate with the severity of knee OA.

Although a number of studies $(7,8,11,13)$ have investigated the differences in serum SA levels between OA patients and healthy controls, to the best of our knowledge, an investigation into the association between SA levels and disease severity in OA has not yet been performed. Therefore, the aim of the present study was to determine the correlation between SA concentrations in the serum and SF and radiographic disease severity in patients with knee OA in order to assess the role of $\mathrm{SA}$ in OA pathophysiology.

\section{Materials and methods}

Patients. A total of 234 patients diagnosed with knee $\mathrm{OA}$, according to the criteria of the American College of Rheumatology (13), were enrolled in the present study. 
Table I. Characteristics of knee OA patients and healthy controls.

\begin{tabular}{lccc}
\hline Characteristics & Knee OA patients $(\mathrm{n}=234)$ & Healthy controls $(\mathrm{n}=160)$ & P-value \\
\hline Age, years & $60.40 \pm 9.06$ & $60.12 \pm 8.34$ & 0.757 \\
Gender, male/female, $\mathrm{n}$ & $86 / 148$ & $63 / 97$ & 0.598 \\
BMI, $\mathrm{kg} / \mathrm{m}^{2}$ & $25.69 \pm 3.28$ & $25.40 \pm 3.12$ & 0.378 \\
Serum SA, mmol/1 & $2.32(1.74-2.72)$ & $1.82(1.49-2.31)$ & $<0.05$ \\
SF SA, mmol/l & $0.67(0.48-0.96)$ & &
\end{tabular}

OA, osteoarthritis; BMI, body mass index; SA, sialic acid; SF, synovial fluid.

Table II. SA levels in the serum and SF of knee OA patients with various KL grades.

\begin{tabular}{llll}
\hline SA, mmol/1 & Grade 2 $(\mathrm{n}=64)$ & Grade 3 $(\mathrm{n}=98)$ & Grade $4(\mathrm{n}=72)$ \\
\hline Serum & $2.26(1.59-2.67)$ & $2.24(1.65-2.81)$ & $2.36(1.98-2.65)$ \\
SF & $0.58(0.40-0.69)$ & $0.67(0.48-1.01)$ & $0.85(0.64-1.17)$ \\
\hline
\end{tabular}

OA, osteoarthritis; SA, sialic acid; SF, synovial fluid; KL, Kellgren-Lawrence. P-values are for all three groups being compared.

Patients that had acute or chronic inflammatory knee disease, rheumatoid arthritis (RA), systemic or autoimmune diseases or previous knee trauma were excluded from the study. The control group consisted of 160 healthy subjects with no clinical or radiological evidence of OA. Furthermore, the study included 20 patients that had suffered a knee injury or fracture, thus, knee SF samples were collected from these individuals. The study was approved by the Ethics Committee of the Beijing Charity Hospital (Beijing, China) and informed consent was provided by all the subjects.

Radiographic assessment of $O A$. Disease severity assessments were performed using the Kellgren-Lawrence (KL) grading system (14). Subjects who had radiographic knee OA of KL grade $\geq 2$ in at least one knee were defined as OA patients. Subjects who had KL grade 0 for both knees were defined as healthy controls. When the patients were affected in both knees, the grading of the worst affected knee was used for analysis.

Laboratory methods. Venous blood samples were collected from all the subjects following overnight fasting. Prior to any OA treatment, SF was obtained from the OA patients that were receiving hyaluronic acid injection treatment for the first time. SA levels in the serum and SF samples were determined using Warren's thiobarbituric acid assay (15).

Statistical analysis. Data are presented as the mean \pm SD or the median (interquartile range). Data normality was analyzed using the Kolmogorov-Smirnov test. The unpaired t-test, Mann-Whitney $U$ test and $\chi^{2}$ test were utilized to analyze the differences in clinical characteristics between the knee OA patients and the healthy controls. The Kruskal-Wallis test was used to compare the differences in SA levels in the serum and SF of the knee OA patients with various KL grades. Statistical significance of the correlation between the SA levels in the serum and SF and disease severity was determined by Spearman's coefficient and multinomial logistic regression analyses. As SA levels were not normally distributed, logarithmic transformed values were used for multiple linear regression analysis. All statistical analyses were performed using SPSS 13.0 for Windows (SPSS, Inc., Chicago, IL, USA) and $\mathrm{P}<0.05$ was considered to indicate a statistically significant difference.

\section{Results}

Baseline clinical parameters. Baseline clinical parameters of the knee OA patients and healthy controls are shown in Table I. There were no significant differences in age, gender or body mass index (BMI) between the two groups.

SA levels in the serum and SF. Patients with knee OA exhibited significantly elevated serum SA levels as compared with the healthy controls (Table I; Fig. 1A; P<0.05). In addition, the SA levels in the SF increased significantly when compared with the knee injury or fracture patients without knee OA (Fig. 1B; $\mathrm{P}<0.01)$.

SA levels in knee OA patients with various KL grades. SA levels in the serum and SF of knee OA patients with various KL grades are shown in Table II. No statistically significant differences in the serum SA levels were identified between the patients with various KL grades (Fig. 2A; P=0.299). However, knee OA patients with KL grade 4 had significantly higher SA levels in the SF when compared with KL grade 2 $(\mathrm{P}<0.01)$ and 3 patients $(\mathrm{P}<0.05$; Fig. $2 \mathrm{~B})$. In addition, higher SA levels in the SF were identified in knee OA patients with KL grade 3 as compared with KL grade 2 OA patients (Fig. 2B). 
A

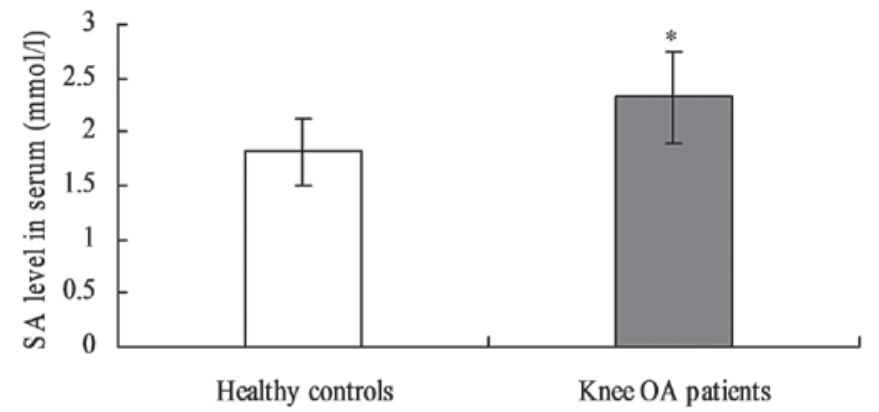

B

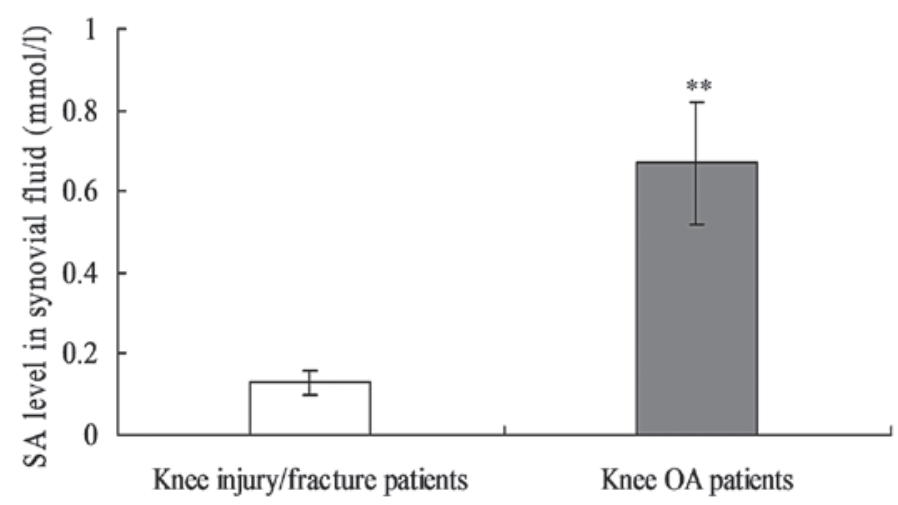

Figure 1. SA levels in the (A) serum and (B) SF of knee OA patients. ${ }^{*} \mathrm{P}<0.05$, vs. healthy controls, ${ }^{* *} \mathrm{P}<0.01$, vs. knee injury/fracture patients. SF, synovial fluid; SA, sialic acid; OA, osteoarthritis.

A

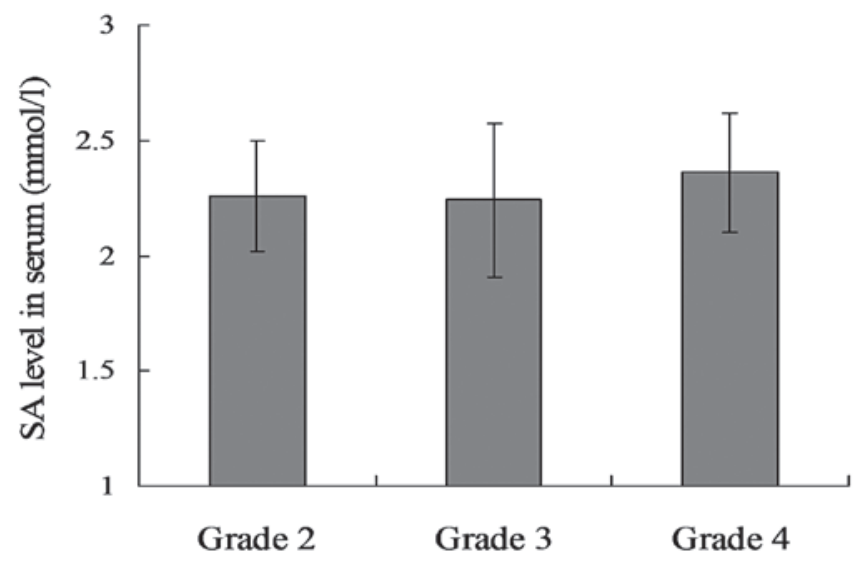

B

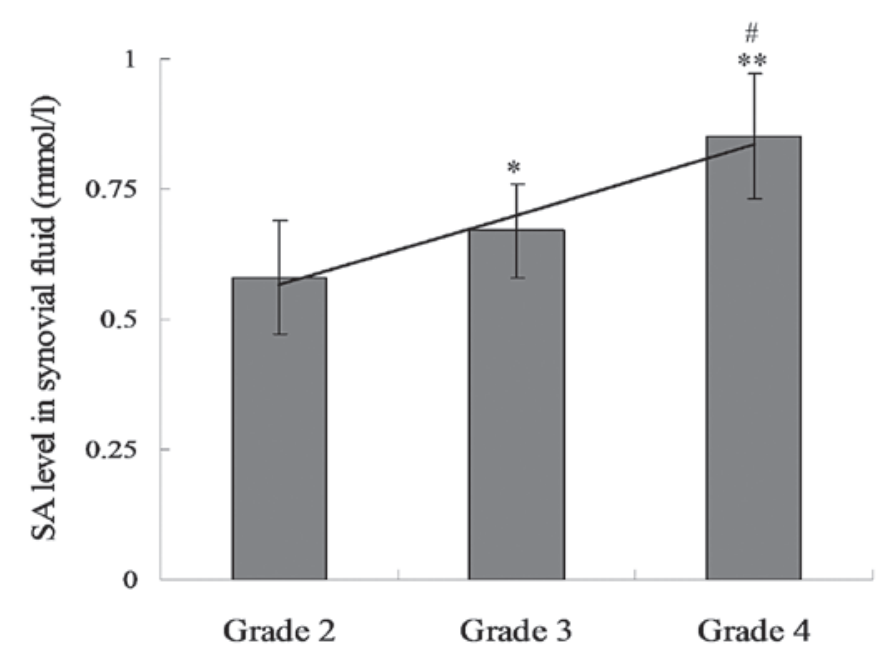

Figure 2. Association between SA levels in the (A) serum and (B) SF with various KL grades. ${ }^{~} \mathrm{P}<0.05$ and ${ }^{* *} \mathrm{P}<0.01$, vs. grade 2 OA patients. ${ }^{*} \mathrm{P}<0.05$, vs. grade 2 OA patients. SF, synovial fluid; SA, sialic acid; OA, osteoarthritis; KL, Kellgren-Lawrence. 
A
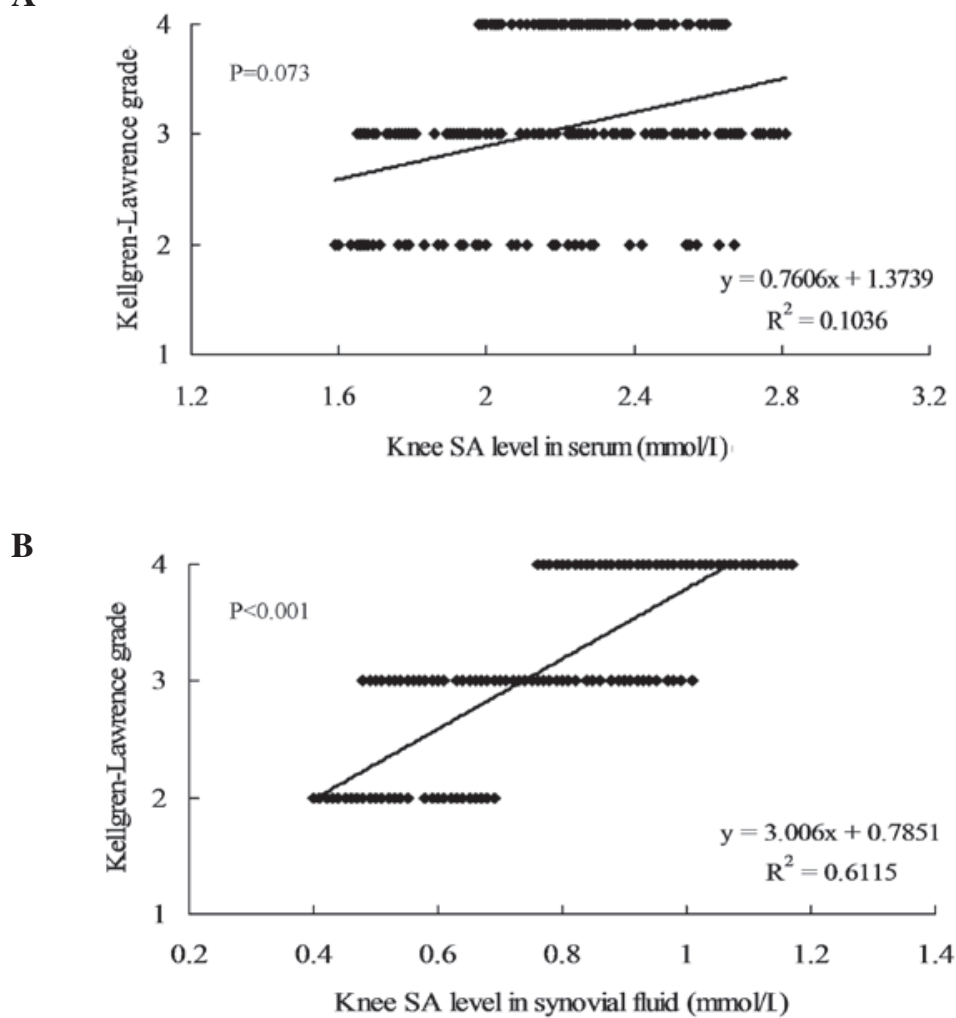

Figure 3. Correlation between KL grades and the SA level in the (A) serum and (B) SF of knee OA patients. SF, synovial fluid; SA, sialic acid; OA, osteoarthritus; KL, Kellgren-Lawrence.

Correlation between clinical parameters and KL grades. Spearman's correlation analysis indicated that the SA levels in the serum did not correlate with KL grades (Fig. 3; r=0.117; $\mathrm{P}=0.073)$. However, there was a significant positive correlation between SA levels in the SF and KL grades (Fig. 3; r=0.353; $\mathrm{P}<0.001)$.

Characteristics, including age, gender, BMI and SA levels in the serum and SF, were then analyzed using a multinomial logistic regression model. Multinomial logistic regression analysis demonstrated that SA levels in the SF positively correlated with $\mathrm{KL}$ grades $(\mathrm{P}<0.001)$. However, there was no significant correlation between the serum levels of SA and $\mathrm{KL}$ grades following multinomial logistic regression analysis ((Fig. 3; $\mathrm{P}=0.103)$.

\section{Discussion}

The results of the present study indicate that patients with knee OA have significantly elevated levels of serum SA compared with healthy controls. In addition, SA levels in the SF positively correlated with KL grades. To the best of our knowledge, this is the first study to demonstrate that SA levels in the SF of knee OA patients correlate with the severity of OA.

$\mathrm{OA}$ is not considered to be a classical inflammatory disease mainly due to the absence of neutrophils in the SF and the lack of systemic manifestations of inflammation. However, OA is associated with symptoms of inflammation, including joint pain, swelling and stiffness, which result in functional impairment and disability (16). It is estimated that $\sim 60 \%$ of joints affected by OA have synovial inflammation at the time of joint replacement (17). Numerous components of the complement cascade and other inflammatory mediators are reported to be elevated in the SF of OA patients (18). Serum SA concentration is elevated during the acute-phase reaction due to an increase in the serum concentration of SA-carrying acute-phase proteins, as well as a higher degree of sialylation of these proteins (19). It has been hypothesized that a single measurement of serum SA may be a useful estimate of the inflammatory status of an individual (20). The results of the present study indicated that serum SA levels were significantly elevated in patients with knee OA as compared with the healthy controls. In addition, SA concentrations in the SF exhibited a significant correlation with KL grades. These observations are consistent with those of a previous study, which demonstrated that OA patients had significantly higher serum SA levels than healthy controls (13). The current results indicate that SA may play an important role in the pathogenesis of OA, thus, SA levels may serve as a new biomarker for predicting the presence and progression of OA.

Serum SA levels have been shown to be elevated in patients with RA, as compared with healthy controls (13). Kosakai also reported that serum SA levels were moderately high in patients with stage 2 or 3 RA. Furthermore, SA levels in the SF were higher in cases of RA and within the normal range in cases of OA (21). The mechanisms of OA and RA are associated with inflammation. Thus, SA appears to contribute to the presence of OA and RA as an inflammatory factor.

There are several limitations of the present study. Firstly, this is a cross-sectional study performed with a relatively small sample size. Therefore, the observations should be vali- 
dated by further longitudinal studies with a larger population sample. Secondly, SA levels in the SF from healthy controls were not assessed due to ethical concerns.

In conclusion, knee OA patients have significantly elevated serum SA levels as compared with healthy controls. In addition, SA levels in the SF positively correlate with the severity of knee OA. Therefore, SA levels in the SF may serve as a new biomarker, in addition to the traditional methods, for assessing the severity of knee OA.

\section{Acknowledgements}

The study was supported by a grant from the Basic Scientific Research Foundation of China Rehabilitation Research Center (no. 2012CZ-9).

\section{References}

1. Kalai E, Bahlous A, Charni N, Bouzid K, Sahli H, Chelly M, Meddeb M, Zouari B, Abdelmoula J and Sellami S: Increased urinary type II collagen C-telopeptide levels in Tunisian patients with knee osteoarthritis. Clin Lab 58: 209-215, 2012.

2. Xu L, Zhu GB, Wang L, Wang DF and Jiang XR: Synovial fluid omentin-1 levels are inversely correlated with radiographic severity of knee osteoarthritis. J Investig Med 60: 583-586, 2012

3. Pantsulaia I, Kalichman L and Kobyliansky E: Association between radiographic hand osteoarthritis and RANKL, OPG and inflammatory markers. Osteoarthritis Cartilage 18: 1448-1453, 2010.

4. Stannus O, Jones G, Cicuttini F, Parameswaran V, Quinn S, Burgess J and Ding C: Circulating levels of IL-6 and TNF- $\alpha$ are associated with knee radiographic osteoarthritis and knee cartilage loss in older adults. Osteoarthritis Cartilage 18: $1441-1447,2010$

5. Wolfe F: The C-reactive protein but not erythrocyte sedimentation rate is associated with clinical severity in patients with osteoarthritis of the knee or hip. J Rheumatol 24: 1486-1488, 1997.

6. Yokoyama H, Jensen JS, Jensen T and Deckert T: Serum sialic acid concentration is elevated in IDDM especially in early diabetic nephropathy. J Intern Med 237: 519-523, 1995.
7. Pönniö M, Alho H, Nikkari ST, Olsson U, Rydberg U and Sillanaukee P: Serum sialic acid in a random sample of the general population. Clin Chem 45: 1842-1849, 1999.

8. Taniuchi K, Chifu K, Hayashi N, Nakamachi Y, Yamaguchi N, Miyamoto Y, Doi K, Baba S, Uchida Y, Tsukada Y and Sugimori T: A new enzymatic method for the determination of sialic acid in serum and its application for a marker of acute phase reactants. Kobe J Med Sci 27: 91-102, 1981.

9. von Versen-Hoeynck FM, Hubel CA, Gallaher MJ, Gammill HS and Powers RW: Plasma levels of inflammatory markers neopterin, sialic acid, and C-reactive protein in pregnancy and preeclampsia. Am J Hypertens 22: 687-692, 2009.

10. Ozben T: Elevated serum and urine sialic acid levels in renal diseases. Ann Clin Biochem 28: 44-48, 1991

11. Bhide SV, Parekh AJ and Desai MP: Sialic acid levels in serum and cerebrospinal fluid (CSF) in infectious and noninfectious conditions. Biochem Med 18: 99-101, 1977.

12. Shamberger RJ: Serum sialic acid in normals and in cancer patients. J Clin Chem Clin Biochem 22: 647-651, 1984.

13. Alturfan AA, Uslu E, Alturfan EE, Hatemi G, Fresko I and Kokoglu E: Increased serum sialic acid levels in primary osteoarthritis and inactive rheumatoid arthritis. Tohoku J Exp Med 213: 241-248, 2007.

14. Kellgren JH and Lawrence JS: Radiological assessment of osteo-arthrosis. Ann Rheum Dis 16: 494-502, 1957.

15. Warren L: The thiobarbituric acid assay of sialic acids. J Biol Chem 234: 1971-1975, 1959.

16. Felson DT: Clinical practice. Osteoarthritis of the knee. N Engl J Med 354: 841-848, 2006.

17. Cooke TD: Immune pathology in polyarticular osteoarthritis. Clin Orthop Relat Res 213: 41-49, 1986.

18. Gobezie R, Kho A, Krastins B, Sarracino DA, Thornhill TS, Chase M, Millett PJ and Lee DM: High abundance synovial fluid proteome: distinct profiles in health and osteoarthritis. Arthritis Res Ther 9: R36, 2007.

19. Chavan MM, Kawle PD and Mehta NG: Increased sialylation and defucosylation of plasma proteins are early events in the acute phase response. Glycobiology 15: 838-848, 2005.

20. Browning LM, Krebs JD and Jebb SA: Discrimination ratio analysis of inflammatory markers: implications for the study of inflammation in chronic disease. Metabolism 53: 899-903, 2004.

21. Kosakai O: Clinical relevance of sialic acids determination in serum and synovial fluid in orthopaedic disorders. Rinsho Byori 39: 197-207, 1991 (In Japanese). 